\title{
An Unusual Case of Constrictive Pericarditis in a Young Patient With Childhood History of Successfully Treated Kawasaki Disease
}

\author{
Obiora Maludum ${ }^{\mathrm{a}, \mathrm{c}}$, Adaeze Ezeume ${ }^{\mathrm{b}}$, Nene Ugoeke ${ }^{\mathrm{a}}$, Dawn Calderon ${ }^{\mathrm{a}}$, Peter Lapman ${ }^{\mathrm{a}}$
}

\begin{abstract}
Kawasaki disease, also known as mucocutaneous lymph node syndrome, is a disease in which blood vessels in the body become inflamed. It occurs usually in childhood and one of the well-known cardiac complications is coronary artery aneurysm which occurs usually after 1 - 2 years. Constrictive pericarditis is not a known sequela of Kawasaki disease. We present a case of a 21 -year-old white male referred to the emergency department by his gastroenterologist after an outpatient abdominal ultrasound obtained for elevated liver enzymes revealed ascites and bilateral pleural effusion. He has background history of Kawasaki disease at the age of 8 years that was treated with aspirin and intravenous immunoglobulin (IVIG) without any cardiac complications based on echocardiogram obtained after treatment. Autoimmune screen was negative. He had echocardiogram which revealed evidence of constrictive pericarditis.
\end{abstract}

Keywords: Constrictive pericarditis; Kawasaki disease; Pericardial disease

\section{Introduction}

Kawasaki disease (KD), also known as mucocutaneous lymph node syndrome, is a disease in which blood vessels in the body become inflamed. It occurs usually in childhood and one of the well-known cardiac complications is coronary artery aneurysm which occurs usually after $1-2$ years. Constrictive pericarditis $(\mathrm{CP})$ occurs when the pericardium becomes fibrotic and thickens to impede normal cardiac function, either systolic or diastolic. It is not known if $\mathrm{CP}$ is a sequela of $\mathrm{KD}$.

Manuscript submitted April 30, 2018, accepted May 15, 2018

${ }^{a}$ Cardiovascular Disease Section, Department of Internal Medicine, Jersey Shore University Medical Center, Neptune, NJ, USA

bDepartment of Internal Medicine, Jersey Shore University Medical Center, Neptune, NJ, USA

${ }^{\mathrm{c} C o r r e s p o n d i n g ~ A u t h o r: ~ O b i o r a ~ M a l u d u m, ~ C a r d i o v a s c u l a r ~ D i s e a s e ~ S e c t i o n, ~}$ Department of Internal Medicine, Jersey Shore University Medical Center, Neptune, NJ 07753, USA. Email: Obimal@yahoo.com

doi: https://doi.org/10.14740/jmc3071w

\section{Case Report}

A 21-year-old white male was referred to the emergency department by his gastroenterologist after an outpatient abdominal ultrasound obtained for elevated liver enzymes revealed ascites and bilateral pleural effusions. He had a background history of KD at the age of 8 years old that was treated with aspirin and intravenous immunoglobulin (IVIG) without any cardiac complications based on echocardiogram obtained after treatment; refractory gastroesophageal reflux disease being managed by a gastroenterologist and methicillin resistant staphylococcus aureus infections in the axilla treated with oral antibiotics with complete resolution. Upon presentation, he was noted to have bilateral lower extremity pitting edema, mildly elevated aspartate amino-transferase $48 \mathrm{IU} / \mathrm{L}$ and alkaline phosphatase $166 \mathrm{IU} / \mathrm{L}$, total bilirubin $3.6 \mathrm{mg} / \mathrm{dL}$, direct bilirubin $1.2 \mathrm{mg} / \mathrm{dL}$, international normalized ratio (INR) 1.61 , creatinine $1.34 \mathrm{mg} / \mathrm{dL}$, with moderate blood on urinalysis. Electrocardiogram (EKG) revealed normal sinus rhythm at 97 beats per minute, low voltage and T-wave inversions in the inferolateral leads similar to previous EKG (Fig. 1). Abdominal ultrasound with Doppler revealed patent arterial and venous blood flow, mild perihepatic ascites and large pleural effusions.

Further investigational tests were ordered as recommended by infectious disease, nephrology, rheumatology, cardiology which included but were not limited to erythrocyte sedimentation rate, C-reactive protein, creatinine, phosphokinase, hepatitis panel, human immunodeficiency virus, complement levels, anti-glomerular membrane antibodies, Ebstein Barr virus (EBV)/cytomegalovirus (CMV)/Coxsackie virus serology, DNAse B antibodies, anti-nucleic acid antibodies, scleroderma antibodies, anti-Smith antibodies, anti-Sjogren's-syndromerelated antigen $\mathrm{A}$ and $\mathrm{B}$ antibodies, Toxoplasma immunoglobulin $\mathrm{G}$ and $\mathrm{M}$, QuantiFERON, C-ANCA, P-ANCA, RNP (ENA), and thyroid stimulating hormone. They were all within normal limits except for elevated EBV immunoglobulin G.

An echocardiogram was obtained which showed left ventricular systolic function is normal with an EF of $60-65 \%$, Borderline pericardial thickening and hyper-echoicity with respiratory variation to mitral inflow and ventricular septal bounce suggesting constrictive pericarditis, with elevated right atrial pressure (Fig. 2a-c).

Patient also underwent thoracentesis with fluid analysis consistent with transudative fluid based on light's criteria, neg- 


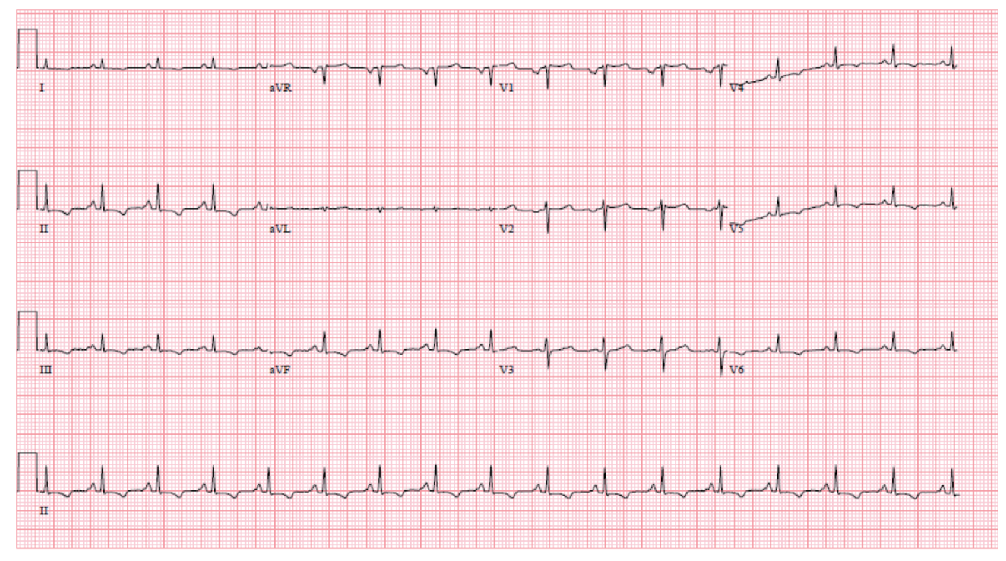

Figure 1. Electrocardiogram showing sinus rhythm, low voltage and inferolateral T wave inversion.
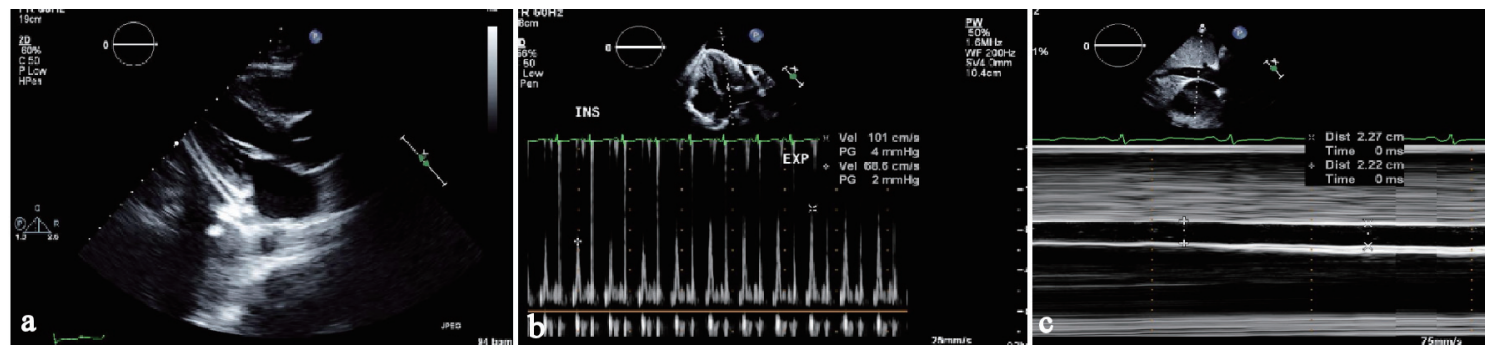

Figure 2. (a) Parasternal long axis view of transthoracic echocardiogram (TTE) showing thickened pericardium in the inferolateral wall. (b) Showing variation on mitral inflow with respiration on TTE. (c) 2D directed M-mode on TTE showing dilated noncollapsing inferior vena cava.

ative for malignant cells. Patient was discharged with the instruction to follow up with cardiology and rheumatology however was lost to follow up due to changes in health insurance.

Patient did follow up with pulmonology who performed repeat bilateral thoracentesis for pleural effusions which were transudative on fluid analysis without malignant cells on cytology. Patient presented once more for recurrent pleural effusion 8 months later during which a repeat echocardiogram was done which showed preserved ejection fraction, findings consistent with constrictive physiology, septal bounce, dilated inferior vena cava with reduced respiratory variability, thickened and calcified pericardium (Fig. 3a-c). Computed tomography (CT) of the chest showed large pleural effusions and mild pericardial thickening (Fig. 4).
Patient was discharged home on torsemide for diuresis and pericardial anti-inflammatory colchicine with the recommendation that he might need a right and left heart cardiac catheterization and surgical pericardiectomy if he continues to retain significant fluid.

\section{Discussion}

$\mathrm{CP}$ results from fibrosis and subsequent loss of pericardial sac elasticity leading to limited movement of the chambers of the heart. It is most commonly idiopathic or caused by viral etiology (42-61\%), following cardiac surgery (11-37\%), radiation (2-31\%), diseases of connective tissue (3-7\%), infectious dis-
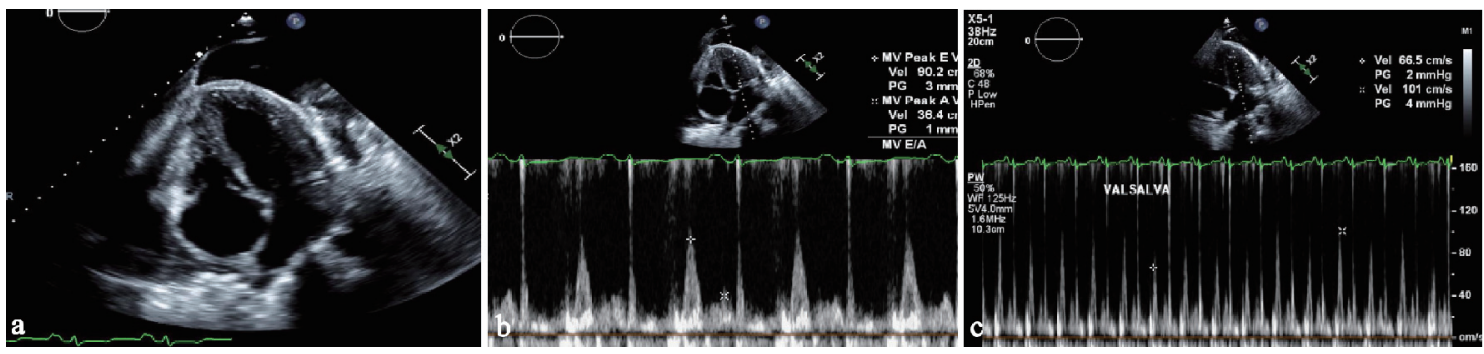

Figure 3. (a) Apical 4-chamber view of TTE showing thickened pericardium. (b) Pulsed wave mitral inflow on TTE showing restrictive filling pattern with E/A of 2.5. (c) Pulsed wave Doppler of mitral inflow on TTE showing variation of inflow with Valsalva maneuver. 


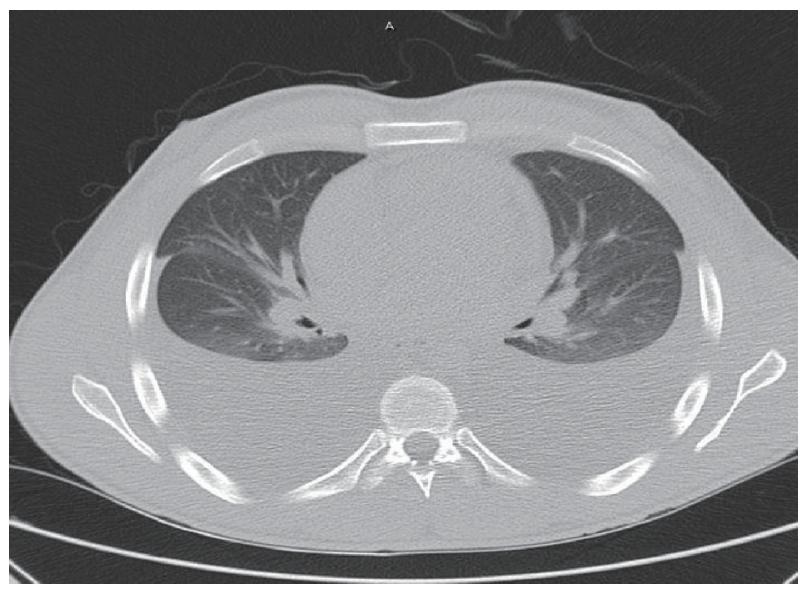

Figure 4. CT chest without contrast showing large bilateral pleural effusions associated with some compressive atelectasis. There appears to be mild diffuse pericardial thickening as opposed to hyperdense pericardial fluid.

eases such as tuberculosis (3-15\%), other categories such as cancer, medications, trauma (1-10\%) [1-2] etc.

Patients usually present with signs and symptoms of right heart failure and decreased cardiac output which include but are not limited to hepatic congestion leading to abdominal discomfort, peripheral edema, hepatojugular reflux, jugular venous distension, jaundice, dyspnea on exertion, pericardial knock, etc.

Initial workup for $\mathrm{CP}$ includes electrocardiogram (EKG), chest radiograph and echocardiogram (ECHO) [3]. One is likely to find nonspecific ST changes, tachycardia and low voltage (as seen in this patient), atrial fibrillation on EKG [4]. A calcified ring around the heart on chest X-ray is most commonly seen in idiopathic causes of pericardial disease and according to a retrospective review is associated with longer lasting symptoms, likelihood of atrial arrhythmias and increased perioperative mortality [5]. Echocardiogram is also useful in the diagnosis of CP. Some of the findings include septal bounce, no changes in venous return during inspiration, dilated inferior vena cava without inspiratory collapse which were all seen in this patient.

KD is vasculitis common in childhood [6] which can result in cardiac complications such as aneurysms of the coronary artery, decreased contractility, myocardial infarction and arrhythmias. The risk of CA aneurysm is decreased with the use of IVIG [7] which this patient received in a timely manner at the age of 8 years old. While KD can result in pericarditis and pericardial effusion in acute setting [8], there has been no direct causal relationship established between KD and CP especially in adults. One poster abstract that was presented at American College of Cardiology Scientific Session in 2017 did describe a case of $\mathrm{CP}$ as chronic sequela of undiagnosed KD [9]. But not much has been published on the association between $\mathrm{KD}$ and $\mathrm{CP}$.

The mainstay treatment of transient $\mathrm{CP}$ is symptomatic medical management with resolution of symptoms in some cases $[3,10]$. In chronic $\mathrm{CP}$ however, pericardiectomy is the only definitive therapy [3]. Early intervention has been shown to improve both early and late functional and survival outcome [11].

This case serves to illustrate the possibility of a new emerging cause of constrictive pericarditis. It also serves as a reminder for physicians and cardiologists to ask about signs and symptoms that might point to a background history of KD when $\mathrm{CP}$ is suspected in a patient presenting with right heart failure.

\section{Conclusions}

This case showed CP in a young patient with history of treated $\mathrm{KD}$. There is limited evidence in the literature on the association between the two diseases. More cases need to be published to establish this association. One should however consider $\mathrm{CP}$ as a sequela of $\mathrm{KD}$ in a young patient with no other etiology for the CP. It is possible that the same mechanism of inflammation in the coronary blood vessel that leads to coronary aneurysm also cause inflammation in the pericardium that leads to $\mathrm{CP}$

\section{Conflict of Interest}

No conflict of interest from any of the authors.

\section{References}

1. Cameron J, Oesterle SN, Baldwin JC, Hancock EW. The etiologic spectrum of constrictive pericarditis. Am Heart J. 1987;113(2 Pt 1):354-360.

2. Ling LH, Oh JK, Schaff HV, Danielson GK, Mahoney DW, Seward JB, Tajik AJ. Constrictive pericarditis in the modern era: evolving clinical spectrum and impact on outcome after pericardiectomy. Circulation. 1999;100(13):1380-1386.

3. Adler Y, Charron P, Imazio M, Badano L, Baron-Esquivias G, Bogaert J, Brucato A, et al. 2015 ESC Guidelines for the diagnosis and management of pericardial diseases: The Task Force for the Diagnosis and Management of Pericardial Diseases of the European Society of Cardiology (ESC)Endorsed by: The European Association for Cardio-Thoracic Surgery (EACTS). Eur Heart J. 2015;36(42):2921-2964.

4. Talreja DR, Edwards WD, Danielson GK, Schaff HV, Tajik AJ, Tazelaar HD, Breen JF, et al. Constrictive pericarditis in 26 patients with histologically normal pericardial thickness. Circulation. 2003;108(15):1852-1857.

5. Ling LH, Oh JK, Breen JF, Schaff HV, Danielson GK, Mahoney DW, Seward JB, et al. Calcific constrictive pericarditis: is it still with us? Ann Intern Med. 2000;132(6):444450.

6. Burns JC, Glode MP. Kawasaki syndrome. Lancet. 2004;364(9433):533-544.

7. Newburger JW, Takahashi M, Gerber MA, Gewitz MH, Tani LY, Burns JC, Shulman ST, et al. Diagnosis, treatment, and long-term management of Kawasaki disease: 
a statement for health professionals from the Committee on Rheumatic Fever, Endocarditis and Kawasaki Disease, Council on Cardiovascular Disease in the Young, American Heart Association. Circulation. 2004;110(17):27472771.

8. Printz BF, Sleeper LA, Newburger JW, Minich LL, Bradley T, Cohen MS, Frank D, et al. Noncoronary cardiac abnormalities are associated with coronary artery dilation and with laboratory inflammatory markers in acute Kawasaki disease. J Am Coll Cardiol. 2011;57(1):86-92.

9. Avula HR, Mendoza D, Chen S, Lundstrom R. Constric- tive pericarditis due to kawasaki disease. JACC (Journal of the American College of Cardiology). 2017;69(11):23602360.

10. Sagrista-Sauleda J, Permanyer-Miralda G, Candell-Riera J, Angel J, Soler-Soler J. Transient cardiac constriction: an unrecognized pattern of evolution in effusive acute idiopathic pericarditis. Am J Cardiol. 1987;59(9):961-966.

11. Depboylu BC, Mootoosamy P, Vistarini N, Testuz A, ElHamamsy I, Cikirikcioglu M. Surgical treatment of constrictive pericarditis. Tex Heart Inst J. 2017;44(2):101106. 